\title{
The Maximal Length of a $k$-Separator Permutation
}

\author{
Benjamin Gunby* \\ Department of Mathematics \\ Massachusetts Institute of Technology \\ Cambridge, Massachusetts, U.S.A. \\ bgunby@mit.edu
}

Submitted: Sep 29, 2013; Accepted: Jul 28, 2014; Published: Aug 6, 2014

Mathematics Subject Classifications: 05A05, 05A20

\begin{abstract}
A permutation $\sigma \in S_{n}$ is a $k$-separator if all of its patterns of length $k$ are distinct. Let $F(k)$ denote the maximal length of a $k$-separator. Hegarty (2013) showed that $k+\lfloor\sqrt{2 k-1}\rfloor-1 \leqslant F(k) \leqslant k+\lfloor\sqrt{2 k-3}\rfloor$, and conjectured that $F(k)=k+\lfloor\sqrt{2 k-1}\rfloor-1$. This paper will strengthen the upper bound to prove the conjecture for all sufficiently large $k$ (in particular, for all $k \geqslant 320801$ ).
\end{abstract}

\section{Introduction and Definitions}

We begin by defining an important and well-known concept: that of pattern containment (specifically, in the case of permutations).

Definition. A permutation $\sigma$ of length $n$ contains a permutation $\pi$ of length $k$ as a pattern if $n \geqslant k$ and there is a length- $k$ subsequence of $\sigma$ with the same relative ordering as $\pi$.

For example, 52413 contains 321 but does not contain 123 .

The concept of pattern containment, especially in the case of permutations, has been studied a great deal in the past forty years, after some results on stack-sortable permutations by Knuth [3]. Originally, the primary focus was on pattern avoidance; i.e. permutations that do not contain a given (fixed) pattern, and related questions, such as the number of such permutations (often considered asymptotically).

*This research was conducted at the University of Minnesota Duluth REU program, supported by NSF/DMS grant 1062709 and NSA grant H98230-11-1-0224. 
In 1999, Arratia [1] introduced the concept of a superpattern-a permutation which contains all permutations of a given length as patterns, and bounded the minimal length of a $k$-superpattern (a bound later improved by Miller [4]).

Recently, Hegarty [2] put forward the idea of a separator permutation-a notion that, in his words, is 'dual to the notion of a superpattern'.

Definition. A permutation $\sigma$ is a $k$-separator if all length- $k$ subsequences of $\sigma$ are orderisomorphic to different length- $k$ permutations.

For example, 2413 is a 3 -separator because it contains $\left(\begin{array}{l}4 \\ 3\end{array}\right)=4$ length-3 subsequences, which correspond to patterns as follows: $241 \rightarrow 231,243 \rightarrow 132,213 \rightarrow 213$, and $413 \rightarrow$ 312. These 4 patterns are all distinct, so 2413 is a 3 -separator.

Definition. We use $F(k)$ to denote the maximum possible length of a $k$-separator.

For example, $F(3)=4$ as 2413 is a 3 -separator of length 4 , but there are no length-5 3 -separators (as $\left(\begin{array}{l}5 \\ 3\end{array}\right)>3$ !).

Hegarty [2] proved that $k+\lfloor\sqrt{2 k-1}\rfloor-1 \leqslant F(k) \leqslant k+\lfloor\sqrt{2 k-3}\rfloor$, showing that $F(k)$ is one of at most two values in all cases. (In fact, the stated theorem replaces $2 k-1$ by $2 k-3$ on the lower bound, but constructions bringing the lower bound to $k+\lfloor\sqrt{2 k-1}\rfloor-1$ are shown later in the paper.) Hegarty also conjectured that, in fact, $F(k)$ equals the lower bound, i.e. $F(k)=k+\lfloor\sqrt{2 k-1}\rfloor-1$ in all cases.

This paper will prove Hegarty's conjecture for all $k \geqslant 320801$.

\section{Hegarty's Lemma and Taxicab Boxes}

We will use as our starting point a lemma of Hegarty (Lemma 2.3 in [2]).

Lemma 2.1. Let $\sigma \in S_{n}$ be a k-separator. Then for any $i \neq j \in\{1, \ldots, n\}$,

$$
\left|\sigma^{-1}(i)-\sigma^{-1}(j)\right|=(n-k+2)+t_{i, j}^{\sigma}-|i-j|,
$$

where $t_{i, j}^{\sigma}$ is the number of integers lying strictly between $i$ and $j$ that also lie between $i$ and $j$ in the permutation $\sigma$.

Since $t_{i, j}^{\sigma} \geqslant 0$, we can substitute $\sigma(i)$ and $\sigma(j)$ for $i$ and $j$ to obtain the following corollary.

Corollary 1. If $\sigma \in S_{n}$ is a $k$-separator and $i \neq j \in\{1, \ldots, n\}$, then

$$
|\sigma(i)-\sigma(j)|+|i-j| \geqslant n-k+2 .
$$

Let $n-k+2=d$. Then the statement $n \leqslant k+\lfloor\sqrt{2 k-1}\rfloor-1$ is equivalent to the statement $n \geqslant \frac{d^{2}}{2}-1$ (given the additional (obvious) assumption $n \geqslant k$ ).

A permutation $\sigma \in S_{n}$ can be interpreted graphically as sending each $i \in\{1, \ldots, n\}$ to the point $(i, \sigma(i))$ in the plane. Corollary 1 can then be interpreted as follows: the taxicab distance between any two such points in a $k$-separator is at least $d$. 
Now, consider the taxicab balls of radius $\frac{d}{2}$ around each such point; these are simply boxes of side length $\frac{d}{\sqrt{2}}$ centered around the point, rotated by $45^{\circ}$. (From now on, we will refer to them interchangeably as 'taxicab balls' and 'boxes'.) By Corollary 1, these cannot overlap. An example for $d=6, n=17=\frac{6^{2}}{2}-1(k=13)$ is shown below; the permutation shown is the 13 -separator $5,10,15,2,7,12,17,4,9,14,1,6,11,16,3,8$, 13.

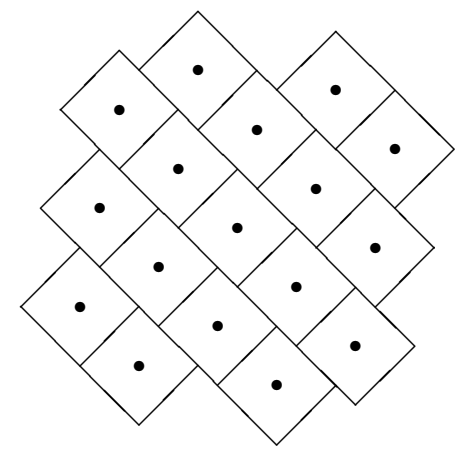

Our results will come from considering the total area of the boxes and the region they must occupy. Since the boxes have radius $\frac{d}{2}$, all points contained in them have $x$ and $y$ coordinates between $1-\frac{d}{2}$ and $n+\frac{d}{2}$, i.e. they are contained in a square of side length $n+d-1$.

In section 3, we will divide this square into several regions, and provide a bound on the area in the regions covered by boxes, given a $k$-separator of sufficiently large size. In particular, the center of the square must be almost entirely covered, while the corners must be mostly empty. Sections 4 and 5 together study the behavior of points in the permutation (i.e. centers of the boxes) that are near the corners of this square. Section 6 uses these points to create a final bound on $d$, and therefore on $k$.

\section{Area Argument}

Each box (taxicab ball) has area $\frac{d^{2}}{2}$, so the total area contained in the boxes is $n \frac{d^{2}}{2}$. We now divide the part of the plane that the boxes can contain into several regions. Namely, we consider four 'strip' areas around the edges, which are given by the equations $|x-1| \leqslant \frac{d}{2},|x-n| \leqslant \frac{d}{2},|y-1| \leqslant \frac{d}{2}$, and $|y-n| \leqslant \frac{d}{2}$. These strips border a square with side length $n-d-1$ that arises from the equation $1+\frac{d}{2} \leqslant x, y \leqslant n-\frac{d}{2}$. Note also that the strips intersect in 4 corner regions, and all of the area of the boxes is contained in the strips and the central square.

We will call the strips $E_{1}, E_{2}, E_{3}$, and $E_{4}$, starting at the bottom and going counterclockwise. We will call the corners $Q_{1}, Q_{2}, Q_{3}$, and $Q_{4}$, starting in the bottom left corner and going counterclockwise (so, for example, $E_{1}$ contains $Q_{1}$ and $Q_{2}$ ). We will call the central square $C$. 


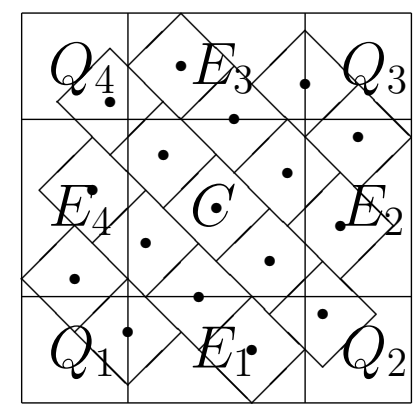

Lemma 3.1. The total area covered by the boxes in each strip $E_{i}, 1 \leqslant i \leqslant 4$ is $\frac{d^{3}+d^{2}}{4}$.

Proof. Without loss of generality, consider only the strip $E_{1}$ (given by the equation $\mid y-$ $1 \mid \leqslant \frac{d}{2}$; the other strips can be argued symmetrically).

Note that since the centers of the boxes correspond to a permutation of $\{1, \ldots, n\}$, there is exactly one box with $y$-coordinate $i$ for all $i \in\{1, \ldots, n\}$. The only boxes that intersect the strip are the ones with centers at $y$-coordinates in $\{1, \ldots, d+1\}$. Furthermore, note that the area of the intersection of the box with center at $y$-coordinate $i$ with the strip plus the area of the intersection for $y$-coordinate $d+1-i$ is $\frac{d^{2}}{2}$, as together the two intersections can be rearranged to form an entire box. Thus the total area of the intersections is $\frac{(d+1) \frac{d^{2}}{2}}{2}=\frac{d^{3}+d^{2}}{4}$.

The total area taken up by the boxes must equal the area of the central square covered by the boxes, plus the area in each strip $E_{i}(1 \leqslant i \leqslant 4)$ covered by the boxes, minus the area in each corner region $Q_{i}(1 \leqslant i \leqslant 4)$ covered by the boxes (as this area is counted twice, as $Q_{i}$ is contained in both $E_{i}$ and $E_{i-1}$, where indices are taken modulo 4). For each $i \in\{1,2,3,4\}$, let $A_{i}$ be the covered area in the corner region $Q_{i}$. Let $A=A_{1}+A_{2}+A_{3}+A_{4}$ be the total covered area in the corner regions, and $B$ be the total uncovered area in the center region $C$.

Noting that $C$ has side length $n-d-1$, and applying Lemma 3.1, we obtain the equality

$$
n \frac{d^{2}}{2}=(n-d-1)^{2}+d^{3}+d^{2}-A-B
$$

We first apply (1) using the trivial bounds $A \geqslant 0$ and $B \geqslant 0$. We obtain $n \frac{d^{2}}{2} \leqslant$ $n^{2}-2 n d+d^{3}+2 d^{2}-2 n+2 d+1$, or rearranging,

$$
n^{2}-n\left(\frac{d^{2}}{2}+2 d+2\right)+\left(d^{3}+2 d^{2}+2 d+1\right) \geqslant 0
$$

Applying the quadratic formula yields that $n$ must not be between the roots

$$
\frac{d^{2}}{4}+d+1 \pm \frac{d}{4} \sqrt{d^{2}-8 d-8}
$$

if $d \geqslant 9$ (and thus $d^{2}-8 d-8>0$ ). 
Hegarty's original result of $n \leqslant k+\sqrt{2 k-3}$ yields $n \geqslant \frac{d^{2}}{2}-d$, and $\frac{d^{2}}{2}-d \geqslant \frac{d^{2}}{4}+d+1$ for $d \geqslant 9$. Thus for $d \geqslant 9$,

$$
n \geqslant \frac{d^{2}}{4}+d+1+\frac{d}{4} \sqrt{d^{2}-8 d-8}
$$

as $n$ is greater than the other root.

Lemma 3.2. For $d \geqslant 30, \frac{d^{2}}{4}+d+1+\frac{d}{4} \sqrt{d^{2}-8 d-8}>\frac{d^{2}-5}{2}$.

Proof. Multiplying by 4 and rearranging, we seek to show $d \sqrt{d^{2}-8 d-8}>d^{2}-4 d-14$. Squaring both sides and moving all terms to one side yields the equivalent inequality $4\left(d^{2}-28 d-49\right)>0$, which holds for $d>14+7 \sqrt{5} \approx 29.7$.

Corollary 2. For $d \geqslant 30$, we have $n>\frac{d^{2}-5}{2}$. Therefore, if $d \geqslant 30$ and $n<\frac{d^{2}}{2}-1$, either $d$ is even and $n=\frac{d^{2}}{2}-2$ or $d$ is odd and $n=\frac{d^{2}}{2}-\frac{3}{2}$.

Recall that $B$ is the total uncovered area in the central region $C$. We have the following lemma in the odd case. The motivation for the lemma is that when $d$ is odd, the corners of the boxes must have one of their coordinates a half-integer and the other an integer, so they cannot 'mesh' completely.

Lemma 3.3. If $d$ is odd, then $B \geqslant \frac{n}{2}-d-\frac{5}{2}$.

Proof. In the diagram below, the large box represents one of the taxicab balls (of radius $\frac{d}{2}$ ) around the points in the permutation; $X, Y$, and $Z$ are taxicab balls of radius $\frac{1}{2}$, positioned as shown.

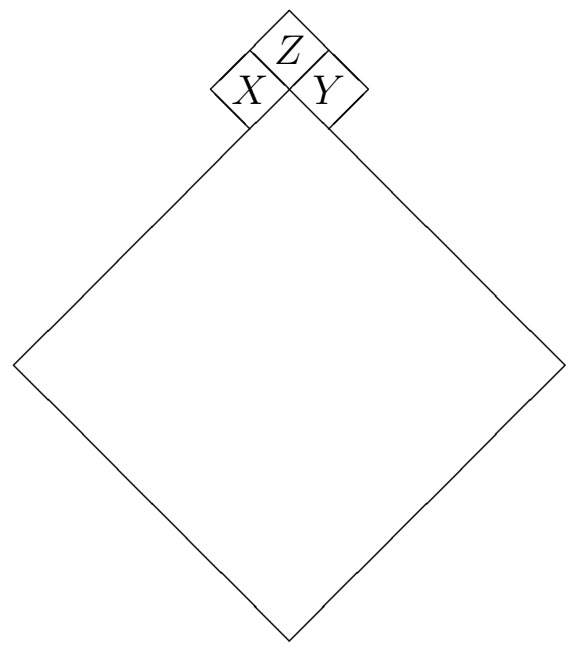

Suppose another one of the taxicab boxes contains $X$. Because the rightmost vertex of $X$ has $y$-coordinate equal to a half-integer (as $d$ is odd), the taxicab box cannot have the rightmost vertex of $X$ as its rightmost vertex. Therefore, it must contain $Z$. 
Similarly, if one of the taxicab boxes contains $Y$, it must contain $Z$. Thus one of $Y$ and $Z$ must be uncovered. It remains to be seen for how many points in the permutation the corresponding boxes $X$ and $Y$ both lie in the inner square.

For $X$ and $Y$ to both lie in the inner square, the $x$-value of the point in the permutation must be between $2+\frac{d}{2}$ and $n-\frac{d}{2}-1$, and the $y$-value must be between $\frac{3}{2}$ and $n-d-\frac{1}{2}$. This eliminates at most $\frac{d+3}{2}+\frac{d+3}{2}+1+(d+1)=2 d+5$ cases (since $d$ is odd), so since $X$ and $Y$ each have area $\frac{1}{2}$, we have $B \geqslant \frac{n-2 d-5}{2}$.

Now, we find a bound on $A$.

Lemma 3.4. If $d \geqslant 30$ and $n<\frac{d^{2}}{2}-1$, then $A \leqslant 6 d+\frac{19}{2}$.

Proof. By Corollary 2, either $d$ is even and $n=\frac{d^{2}}{2}-2$ or $d$ is odd and $n=\frac{d^{2}-3}{2}$. We now apply (1) in both cases.

Case 1. $d$ is even, $n=\frac{d^{2}}{2}-2$.

In this case, simply use (1) with the inequality $B \geqslant 0$. We obtain

$$
\left(\frac{d^{2}}{2}-2\right) \frac{d^{2}}{2} \leqslant\left(\frac{d^{2}}{2}-d-3\right)^{2}+d^{3}+d^{2}-A,
$$

or $A \leqslant 6 d+9<6 d+\frac{19}{2}$.

Case 2. $d$ is odd, $n=\frac{d^{2}}{2}-\frac{3}{2}$.

We now must also use Lemma 3.3. (1) now yields

$$
\left(\frac{d^{2}}{2}-\frac{3}{2}\right) \frac{d^{2}}{2}=\left(\frac{d^{2}}{2}-d-\frac{5}{2}\right)^{2}+d^{3}+d^{2}-A-B
$$

or $A+B=\frac{d^{2}}{4}+5 d+\frac{25}{4}$. By Lemma $3.3, B \geqslant \frac{n}{2}-d-\frac{5}{2}=\frac{d^{2}}{4}-d-\frac{13}{4}$, so $A \leqslant$ $\frac{d^{2}}{4}+5 d+\frac{25}{4}-B \leqslant 6 d+\frac{19}{2}$.

This is quite a strong result, as it means that for large values of $d$, almost none of the corner area is covered by boxes, as $6 d+\frac{19}{2}$ is linear in $d$, whereas the area of the $Q_{i}$ is quadratic in $d$.

\section{Improvement on another Lemma of Hegarty}

To bound the corner areas, we must first improve another lemma of Hegarty (Lemma 2.5 in [2]), which states the following.

Lemma 4.1. Let $m \in \mathbb{N}$ and $a_{1} a_{2} \cdots a_{m}$ be any permutation of the integers in $\{1, \ldots, m\}$. Then

$$
\sum_{i=1}^{m-1}\left|a_{i}-a_{i+1}\right| \leqslant \frac{m^{2}-1}{2} .
$$


We will prove the following refined version of the lemma.

Lemma 4.2. Let $m \in \mathbb{N}$ and $a_{1} a_{2} \cdots a_{m}$ be any permutation of the integers in $\{1, \ldots, m\}$. Then

$$
\sum_{i=1}^{m-1}\left|a_{i}-a_{i+1}\right| \leqslant \frac{m^{2}}{2}-\left|a_{1}-\frac{m+1}{2}\right|-\left|a_{m}-\frac{m+1}{2}\right| .
$$

Proof. We only need to show the lemma for 2 distinct cases (since we can replace each $a_{i}$ by $m+1-a_{i}$ if necessary); the case when $a_{1}, a_{m} \geqslant \frac{m+1}{2}$ and the case when $a_{1} \geqslant \frac{m+1}{2} \geqslant a_{m}$. Case 1. $a_{1}, a_{m} \geqslant \frac{m+1}{2}$.

We proceed analogously to Hegarty's proof of Lemma 4.1; that is, let $r$ be the number of $i \in\{1, \ldots, m-1\}$ such that $a_{i}<a_{i+1}$, and $s$ be the number of $i \in\{1, \ldots, m-1\}$ such that $a_{i}>a_{i+1}$. Let $i_{1}, \ldots, i_{r}$ be the indices such that $a_{i_{j}}<a_{i_{j}+1}$. Then all $a_{i_{j}}$ must be distinct and all $a_{i_{j+1}}$ must be distinct. By the definition of the $i_{j}, \sum_{j=1}^{r}\left|a_{i_{j}}-a_{i_{j+1}}\right|=$ $\sum_{j=1}^{r} a_{i_{j+1}}-\sum_{j=1}^{r} a_{i_{j}}$. The latter sum is at least $1+\cdots+r$, and the former is at most $m+\cdots+(m-r)-a_{1}$, since $a_{1}$ cannot be part of the sum. Thus

$$
\sum_{j=1}^{r}\left|a_{i_{j}}-a_{i_{j+1}}\right| \leqslant m(r+1)-r(r+1)-a_{1} .
$$

Similarly, if $i_{1}^{\prime}, \ldots, i_{s}^{\prime}$ are the indices for which $a_{i_{j}^{\prime}}>a_{i_{j}^{\prime}+1}$, then

$$
\sum_{j=1}^{s}\left|a_{i_{j}^{\prime}}-a_{i_{j}^{\prime}+1}\right| \leqslant m(s+1)-s(s+1)-a_{m} .
$$

Adding these, we obtain $\sum_{i=1}^{m-1}\left|a_{i}-a_{i+1}\right| \leqslant m(r+s+2)-r-s-r^{2}-s^{2}-a_{1}-a_{m}$. Applying the condition $r+s=m-1$, the right hand side is maximized when $r=s=\frac{m-1}{2}$, so we obtain

$$
\begin{aligned}
\sum_{i=1}^{m-1}\left|a_{i}-a_{i+1}\right| & \leqslant m(m+1)-\frac{m^{2}-1}{2}-a_{1}-a_{m} \\
& =\frac{m^{2}+2 m+1}{2}-a_{1}-a_{m} \\
& =\frac{m^{2}-1}{2}-\left(a_{1}-\frac{m+1}{2}\right)-\left(a_{m}-\frac{m+1}{2}\right) \\
& =\frac{m^{2}-1}{2}-\left|a_{1}-\frac{m+1}{2}\right|-\left|a_{m}-\frac{m+1}{2}\right|,
\end{aligned}
$$

as desired. 
Case 2. $a_{1} \geqslant \frac{m+1}{2} \geqslant a_{m}$.

As before, we have $\sum_{j=1}^{r}\left|a_{i_{j}}-a_{i_{j}+1}\right|=\sum_{j=1}^{r} a_{i_{j}+1}-\sum_{j=1}^{r} a_{i_{j}}$. However, in this case, not only can $a_{1}$ not appear in the first sum, but $a_{m}$ cannot appear in the second sum. Thus $\sum_{j=1}^{r}\left|a_{i_{j}}-a_{i_{j}+1}\right| \leqslant m+\cdots+(m-r)-a_{1}-(1+\cdots+(r+1))+a_{m}=m(r+1)-(r+1)^{2}-a_{1}+a_{m}$. The other sum has no restrictions and thus is bounded by $m+\cdots+(m-s+1)-(1+\cdots+s)=$ $m s-s^{2}$. Therefore, the sum is at most $m(r+s+1)-(r+1)^{2}-s^{2}-a_{1}+a_{m}$, which is maximized when $r+1=s=\frac{m}{2}$, giving

$$
\begin{aligned}
\sum_{i=1}^{m-1}\left|a_{i}-a_{i+1}\right| & \leqslant \frac{m^{2}}{2}-a_{1}+a_{m} \\
& =\frac{m^{2}}{2}-\left(a_{1}-\frac{m+1}{2}\right)-\left(\frac{m+1}{2}-a_{m}\right) \\
& =\frac{m^{2}}{2}-\left|a_{1}-\frac{m+1}{2}\right|-\left|a_{m}-\frac{m+1}{2}\right| .
\end{aligned}
$$

\section{Bounding Corner Points}

Armed with the previous lemma, we now turn our attention to the area in the corners. Roughly, in this section we will show that each 'corner' $((1,1),(n, 1),(n, n)$, or $(1, n))$ has two points near it, in somewhat specific locations (the possible error in their locations is of order lower than $d$ ).

Assume there is a construction satisfying $n<\frac{d^{2}}{2}-1$.

Recall that $A_{1}, A_{2}, A_{3}$, and $A_{4}$ are the amount of covered area in $Q_{1}, Q_{2}, Q_{3}$, and $Q_{4}$, respectively.

Note that if a box contributes to $A_{1}$ (i.e. part of its area covers part of the region $|x-1| \leqslant \frac{d}{2},|y-1| \leqslant \frac{d}{2}$ ) then both its $x$ - and $y$-coordinates must be at most $d$. (When referring to the coordinates of one of the boxes, we mean the coordinates of its center.)

Therefore, it is logical to consider boxes at $y$-coordinates in $\{1, \ldots, d\}$. In particular, let $x_{1}, \ldots, x_{d}$ be such that $\sigma\left(\left\{x_{1}, \ldots, x_{d}\right\}\right)=\{1, \ldots, d\}$ and $x_{1}<x_{2}<\cdots<x_{d}$ (that is, they are the $x$-coordinates corresponding to the $y$-coordinates 1 through $d$, arranged from left to right). Let $\sigma\left(x_{i}\right)=y_{i}$ for $1 \leqslant i \leqslant d$, so the $y_{i}$ are a permutation of $\{1, \ldots, d\}$.

We apply Lemma 4.2. The idea is that Lemma 4.2 requires the vertical distances $\left|y_{i}-y_{i+1}\right|$ to not be too large, which forces the horizontal distances $\left|x_{i}-x_{i+1}\right|$ to be large, forcing the outer points into the corners. In particular, we obtain

$$
\sum_{i=1}^{d-1}\left|y_{i+1}-y_{i}\right| \leqslant \frac{d^{2}}{2}-\left|y_{1}-\frac{d+1}{2}\right|-\left|y_{d}-\frac{d+1}{2}\right| .
$$

Since $\left|x_{i+1}-x_{i}\right|+\left|y_{i+1}-y_{i}\right| \geqslant d$ by Lemma 2.1 and $x_{i+1}>x_{i}$ by assumption, we must 
have $x_{d}-x_{1} \geqslant d(d-1)-\left(\frac{d^{2}}{2}-\left|y_{1}-\frac{d+1}{2}\right|-\left|y_{d}-\frac{d+1}{2}\right|\right)$, so

$$
x_{d}-x_{1} \geqslant \frac{d^{2}}{2}-d+\left|y_{1}-\frac{d+1}{2}\right|+\left|y_{d}-\frac{d+1}{2}\right| \text {. }
$$

Now suppose that $n \leqslant \frac{d^{2}-3}{2}$. Then $x_{d}-x_{1} \geqslant n-d+\frac{3}{2}+\left|y_{1}-\frac{d+1}{2}\right|+\left|y_{d}-\frac{d+1}{2}\right|$. Rearranging, we get $\left(n-x_{d}\right)+\left|y_{d}-\frac{d+1}{2}\right|+\left(x_{1}-1\right)+\left|y_{1}-\frac{d+1}{2}\right| \leqslant d-\frac{5}{2}$. Thus the taxicab distance from $\left(x_{1}, y_{1}\right)$ to $\left(1, \frac{d+1}{2}\right)$ plus the taxicab distance from $\left(x_{d}, y_{d}\right)$ to $\left(n, \frac{d+1}{2}\right)$ is at most $d-\frac{5}{2}$. Call these two taxicab distances $T C_{1}$ and $T C_{d}$, respectively.

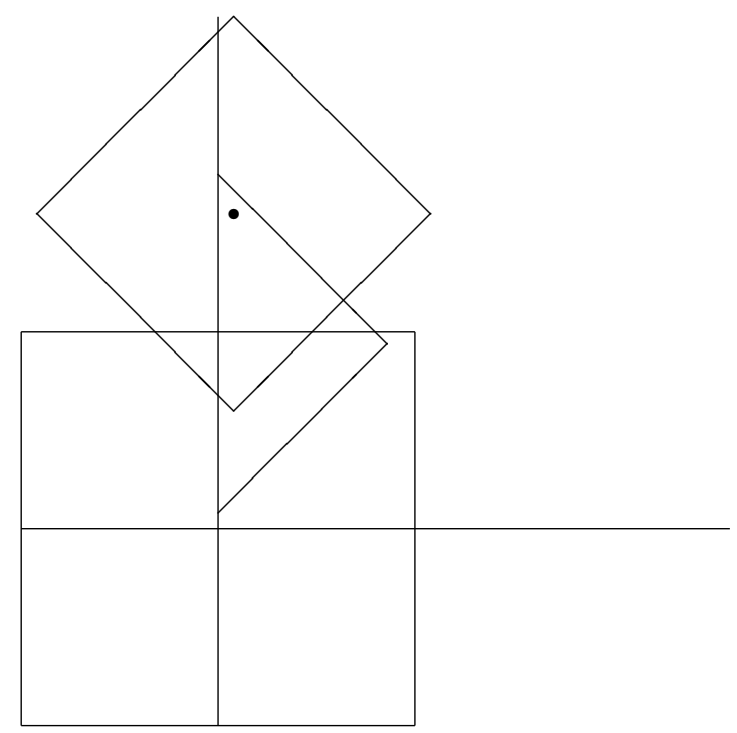

Consider the point $\left(x_{1}, y_{1}\right)$ and the box centered around it, as shown.

Lemma 5.1. If $d \geqslant 50, T C_{1} \geqslant \frac{d+1}{2}-\sqrt{A_{1}}$.

Proof. Suppose that $T C_{1}<\frac{d+1}{2}-\sqrt{A_{1}}$. Then $y_{1}<d+1-\sqrt{A_{1}}$, so the bottom tip of the box lies below $y$-coordinate $\frac{d}{2}+1-\sqrt{A_{1}}$. Thus, more than $A_{1}$ of the area in the box lies in the region $y \leqslant \frac{d}{2}+1$. So some of this area must be outside the corner area, i.e. have $x$-coordinate greater than $\frac{d}{2}+1$ (since the $y$-coordinate is known to be less than that). This implies that $x_{1}+\sqrt{A_{1}}>\frac{d}{2}+1$. But since $T C_{1}<\frac{d+1}{2}-\sqrt{A_{1}}$, we have that $\left|y_{1}-\frac{d+1}{2}\right|<\frac{d+1}{2}-\frac{d}{2}-\sqrt{A_{1}}+\sqrt{A_{1}}=\frac{1}{2}$. Since $y_{1}$ is an integer, $y_{1}=\frac{d+1}{2}$. This, however, implies that the entire bottom-left fourth (and slightly extra) of the box lies in the corner region, so $\frac{d^{2}}{8}<A_{1} \leqslant 6 d+\frac{19}{2}$ (if $d \geqslant 30$ ), so $d^{2}-48 d-76<0$. Thus $d \leqslant 49$, proving the lemma.

Corollary 3. If $d \geqslant 50, \frac{d+1}{2}-\sqrt{A_{1}} \leqslant T C_{1} \leqslant \frac{d}{2}-3+\sqrt{A_{2}}$

Proof. By the lemma we have $T C_{1} \geqslant \frac{d+1}{2}-\sqrt{A_{1}}$. Similarly, $T C_{d} \geqslant \frac{d+1}{2}-\sqrt{A_{2}}$. Since $T C_{1}+T C_{d} \leqslant d-\frac{5}{2}$, we obtain the remaining inequality $T C_{1} \leqslant \frac{d}{2}-3+\sqrt{A_{2}}$.

Lemma 5.2. If $d \geqslant 128, y_{1} \geqslant d+1-\sqrt{A_{1}}$. 
Proof. Suppose $y_{1}<d+1-\sqrt{A_{1}}$. The area of the portion of the box around $\left(x_{1}, y_{1}\right)$ with $y$-coordinate at most $y_{1}-\frac{d}{2}+\sqrt{A_{1}}$ is $A_{1}$. Since $y_{1}<d+1-\sqrt{A_{1}}$, this area has $y$-coordinate less than $\frac{d}{2}+1$, so it is in the bottom strip region. If it is all in the corner region, then we will have the area being strictly greater than $A_{1}$ (because the assumed inequality on $y_{1}$ is strict, there will be additional area above the bottom tip of the box), a contradiction. Therefore, we must have $x_{1}+\sqrt{A_{1}}>\frac{d}{2}+1$, or $x_{1}>\frac{d}{2}+1-\sqrt{A_{1}}$. Since $T C_{1} \leqslant \frac{d}{2}-3+\sqrt{A_{2}}$, this implies $\left|y_{1}-\frac{d+1}{2}\right|<\sqrt{A_{1}}+\sqrt{A_{2}}-3$, so $y_{1}<\frac{d}{2}+\sqrt{A_{1}}+\sqrt{A_{2}}-\frac{5}{2}$. Thus the area in the box around $\left(x_{1}, y_{1}\right)$ that is in the lower strip $\left(\left|y_{1}-1\right| \leqslant \frac{d}{2}\right)$ is greater than $\left(\frac{d}{2}+\frac{7}{2}-\sqrt{A_{1}}-\sqrt{A_{2}}\right)^{2}$. Since $x<\frac{d}{2}+1$, more than half of this area is in the corner region, so $\left(\frac{d}{2}+\frac{7}{2}-\sqrt{A_{1}}-\sqrt{A_{2}}\right)^{2}<2 A_{1}$. Therefore,

$$
(2+2 \sqrt{2}) \sqrt{A_{1}}+2 \sqrt{A_{2}}>d+7 .
$$

Now,

$$
\begin{aligned}
(2+2 \sqrt{2}) \sqrt{A_{1}}+2 \sqrt{A_{2}} & =(2+2 \sqrt{2})^{2} \sqrt{\frac{A_{1}}{(2+2 \sqrt{2})^{2}}}+2^{2} \sqrt{\frac{A_{2}}{2^{2}}} \\
& \leqslant \sqrt{(2+2 \sqrt{2})^{2}+2^{2}} \sqrt{(2+2 \sqrt{2})^{2} \frac{A_{1}}{(2+2 \sqrt{2})^{2}}+2^{2} \frac{A_{2}}{2^{2}}} \\
& =\sqrt{(2+2 \sqrt{2})^{2}+2^{2}} \sqrt{A_{1}+A_{2}} \\
& \leqslant \sqrt{12+8 \sqrt{2}} \sqrt{6 d+\frac{19}{2}},
\end{aligned}
$$

where the first inequality is by Weighted Power Mean and the second by Lemma 3.4. Thus,

$$
d+7<\sqrt{(12+8 \sqrt{2})\left(6 d+\frac{19}{2}\right)} .
$$

Squaring, we obtain $d^{2}+14 d+49<(12+8 \sqrt{2})\left(6 d+\frac{19}{2}\right)=(72+48 \sqrt{2}) d+(114+76 \sqrt{2})$, so $d^{2}-(58+48 \sqrt{2}) d-(65+78 \sqrt{2})>0$. Therefore, $d \leqslant 127$, as desired.

Corollary 4. If $d \geqslant 128, x_{1} \leqslant \sqrt{A_{1}}+\sqrt{A_{2}}-\frac{5}{2}$.

Proof. By Corollary $3, T C_{1} \leqslant \frac{d}{2}-3+\sqrt{A_{2}}$. Since $y_{1} \geqslant d+1-\sqrt{A_{1}}$, we have $\left|y_{1}-\frac{d+1}{2}\right| \geqslant$ $\frac{d+1}{2}-\sqrt{A_{1}}$, so $\left|x_{1}-1\right|=T C_{1}-\left|y_{1}-\frac{d+1}{2}\right| \leqslant \sqrt{A_{1}}+\sqrt{A_{2}}-\frac{7}{2}$. The conclusion follows.

Lemma 5.2 and its corollary together show that if $d \geqslant 128$ there is a point $(x, y)$ in the permutation (i.e. $\sigma(x)=y$ ) such that $x \leqslant \sqrt{A_{1}}+\sqrt{A_{2}}-\frac{5}{2}$ and $d+1-\sqrt{A_{1}} \leqslant y \leqslant d$.

Symmetrically, there must be a point $(x, y)$ in the permutation such that $d+1-$ $\sqrt{A_{1}} \leqslant x \leqslant d$ and $y \leqslant \sqrt{A_{1}}+\sqrt{A_{4}}-\frac{5}{2}$. Now, if these two points are the same, then we must have $d+1-\sqrt{A_{1}} \leqslant \sqrt{A_{1}}+\sqrt{A_{2}}-\frac{5}{2}$, so $d+\frac{7}{2} \leqslant 2 \sqrt{A_{1}}+\sqrt{A_{2}}$. However, $2 \sqrt{A_{1}}+\sqrt{A_{2}}<3 \sqrt{6 d+\frac{19}{2}}$, so $\left(d+\frac{7}{2}\right)^{2}=d^{2}+7 d+\frac{49}{4}<54 d+\frac{171}{2}$, a contradiction with the assumption that $d \geqslant 128$. Thus the points are distinct. 


\section{Resulting Additional Bounds}

In this section, we will use the two points that we showed exist in the previous section, in order to show that area is now forced into the center.

The diagram below shows the two points, along with the boxes around them. (As shown in the diagram, one point must be strictly above and strictly to the left of the other.)

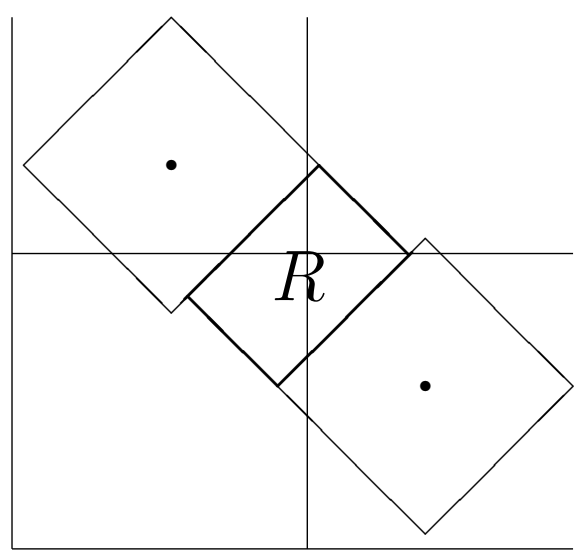

Note that none of the region $R$ can be covered by any other box. This is because its taxicab width is less than $d$ (that is, there exist two points on opposite sides such that the taxicab distance between them is less than $d$ ); in fact, it is equal to the taxicab distance between the two points in the diagram minus $d$, and both points must have both coordinates between 1 and $d$ (inclusive), by their construction. Therefore, if a box covers any point in $R$, it must intersect one of the two boxes already there, which is impossible.

Note that some of $R$ intersects the central area, which creates a new lower bound on $B$.

Lemma 6.1. If $d \geqslant 128, B \geqslant \frac{(d+7)^{2}}{2}-6(d+7) \sqrt{A}+2 A$.

Proof. Call the points $\left(x_{1}, y_{1}\right)$ and $\left(x_{2}, y_{2}\right)\left(\left(x_{1}, y_{1}\right)\right.$ is the upper-left one). We know that $x_{1} \leqslant \sqrt{A_{1}}+\sqrt{A_{2}}-\frac{5}{2}$ by Corollary 4 and $d+1-\sqrt{A_{1}} \leqslant y_{1}$ by Lemma 5.2. We consider the lower intersection of the box around $\left(x_{1}, y_{1}\right)$ with the line $x=\frac{d}{2}+1$. Note that $\left|x-x_{1}\right| \geqslant \frac{d}{2}-\sqrt{A_{1}}-\sqrt{A_{2}}+\frac{7}{2}$. Therefore, $\left|y-y_{1}\right|=\frac{d}{2}-\left|x-x_{1}\right| \leqslant \sqrt{A_{1}}+\sqrt{A_{2}}-\frac{7}{2}$. Thus $y \geqslant y_{1}-\sqrt{A_{1}}-\sqrt{A_{2}}+\frac{7}{2} \geqslant d-2 \sqrt{A_{1}}-\sqrt{A_{2}}+\frac{9}{2}$.

Similarly, the leftmost intersection $(x, y)$ of the box around $x_{2}, y_{2}$ with the line $y=\frac{d}{2}+1$ has $x \geqslant d-2 \sqrt{A_{1}}-\sqrt{A_{4}}+\frac{9}{2}$.

Thus, the triangle $T_{1}$ formed by the 3 points $\left(\frac{d}{2}+1, \frac{d}{2}+1\right),\left(d-2 \sqrt{A_{1}}-\sqrt{A_{4}}+\frac{9}{2}, \frac{d}{2}+1\right)$, and $\left(\frac{d}{2}+1, d-2 \sqrt{A_{1}}-\sqrt{A_{2}}+\frac{9}{2}\right)$ is contained in both $R$ and the central region, and has area

$$
\frac{1}{2}\left(d-2 \sqrt{A_{1}}-\sqrt{A_{4}}+\frac{9}{2}-\left(\frac{d}{2}+1\right)\right)\left(d-2 \sqrt{A_{1}}-\sqrt{A_{2}}+\frac{9}{2}-\left(\frac{d}{2}+1\right)\right) .
$$


Simplifying, we get

$$
\begin{aligned}
& \frac{1}{2}\left(\frac{d}{2}-2 \sqrt{A_{1}}-\sqrt{A_{4}}+\frac{7}{2}\right)\left(\frac{d}{2}-2 \sqrt{A_{1}}-\sqrt{A_{2}}+\frac{7}{2}\right) \\
&= \frac{1}{2}\left(\left(\frac{d+7}{2}\right)^{2}-2\left(\frac{d+7}{2}\right)\left(4 \sqrt{A_{1}}+\sqrt{A_{2}}+\sqrt{A_{4}}\right)\right. \\
&\left.+4 A_{1}+2 \sqrt{A_{1} A_{2}}+2 \sqrt{A_{1} A_{4}}+\sqrt{A_{2} A_{4}}\right) .
\end{aligned}
$$

Since this occurs at every corner and using $A_{1}+A_{2}+A_{3}+A_{4}=A$, we obtain

$$
\begin{aligned}
B & \geqslant \frac{(d+7)^{2}}{2}-3(d+7)\left(\sum_{c y c} \sqrt{A_{1}}\right)+2\left(\sum_{c y c} A_{1}\right) \\
& +2\left(\sum_{c y c} \sqrt{A_{1} A_{2}}\right)+\sqrt{A_{1} A_{3}}+\sqrt{A_{2} A_{4}} \\
& \geqslant \frac{(d+7)^{2}}{2}-3(d+7)\left(\sum_{c y c} \sqrt{A_{1}}\right)+2\left(\sum_{c y c} A_{1}\right) \\
& \geqslant \frac{(d+7)^{2}}{2}-3(d+7)\left(\sum_{c y c} \sqrt{\frac{A}{4}}\right)+2 A \\
& =\frac{(d+7)^{2}}{2}-6(d+7) \sqrt{A}+2 A .
\end{aligned}
$$

Here the last inequality comes from the concavity of the square root function.

We split the remainder of the argument into two cases, depending on the parity of $d$. Case 1. First, assume $d$ is even (and, as before, $d \geqslant 128$ ). Then by the argument in Lemma 3.4, $A+B \leqslant 6 d+9$. Thus

$$
\frac{(d+7)^{2}}{2}-6(d+7) \sqrt{A}+3 A \leqslant 6 d+9
$$

The derivative of this with respect to $A$ is $-\frac{3(d+7)}{\sqrt{A}}+3$, which is negative for $A<(d+7)^{2}$. However, $(d+7)^{2}>d^{2}>6 d+9 \geqslant A+B \geqslant A$ for $d \geqslant 128$, so the derivative is negative. Thus the expression is minimized when $A$ is maximized. Since $A \leqslant 6 d+9$, we must have

$$
\frac{(d+7)^{2}}{2}-6(d+7) \sqrt{6 d+9}+3(6 d+9) \leqslant 6 d+9
$$

which simplifies to

$$
d^{2}+38 d+85 \leqslant 12(d+7) \sqrt{6 d+9} .
$$

Squaring and subtracting, we obtain

$$
d^{4}-788 d^{3}-11778 d^{2}-54020 d-56279 \leqslant 0,
$$

so $d \leqslant 802$. 
Case 2. Now, consider the case when $d$ is odd. This case is somewhat more nuanced, because our previous results already required bounding $B$ below.

The idea is that the uncovered space that causes each of the bounds (Lemma 3.3 and Lemma 6.1) only intersect a relatively small amount, so they can nearly be added directly.

Consider the diagram for Lemma 3.3. Note that the small boxes $X$ and $Y$ are each contained within taxicab radius 1 from the topmost vertex of the box. Since any two such topmost vertices must have taxicab distance at least $d$ between them, there must be at least taxicab distance $d-2$ between (any two points in) any two small boxes (i.e. $X$ or $Y$ ) corresponding to different vertices. But the maximum taxicab distance between any two points in $T_{1}$ is

$$
\left(\frac{d}{2}-2 \sqrt{A_{1}}-\sqrt{A_{4}}+\frac{7}{2}\right)+\left(\frac{d}{2}-2 \sqrt{A_{1}}-\sqrt{A_{2}}+\frac{7}{2}\right)=d-4 \sqrt{A_{1}}-\sqrt{A_{2}}-\sqrt{A_{4}}+7 .
$$

If $4 \sqrt{A_{1}}+\sqrt{A_{2}}+\sqrt{A_{4}}>9$, then the taxicab distance is less than $d-2$, meaning that there can only be at most 1 small box touching $T_{1}$. Otherwise, we can split the triangle along its median from $\left(\frac{d}{2}+1, \frac{d}{2}+1\right)$, and then any two points in $T_{1}$ on the same side of the median must have taxicab distance at most $\frac{d}{2}+\frac{7}{2}<d-2$ for $d>11$, so there are at most two boxes that intersect the region.

Since there are at most two small boxes that intersect $T_{1}$ or any one of the four such triangles, each with area $\frac{1}{2}$, we can add our two bounds for $B$ (the above bound for the even case and Lemma 3.3) while subtracting their maximum intersection, which is thus 4. Therefore

$$
B \geqslant\left(\frac{(d+7)^{2}}{2}-6(d+7) \sqrt{A}+2 A\right)+\left(\frac{d^{2}-3}{4}-d-\frac{5}{2}\right)-4 .
$$

We know from Case 2 of the proof of Lemma 3.4 that $A+B \leqslant \frac{d^{2}}{4}+5 d+\frac{25}{4}$. Thus

$$
\frac{d^{2}}{4}+5 d+\frac{25}{4} \geqslant A+B \geqslant\left(\frac{(d+7)^{2}}{2}-6(d+7) \sqrt{A}+3 A\right)+\left(\frac{d^{2}-29}{4}-d\right),
$$

SO

$$
\left(\frac{(d+7)^{2}}{2}-6(d+7) \sqrt{A}+3 A\right)-6 d-\frac{27}{2} \leqslant 0 .
$$

As in the even case, this is minimized when $A=6 d+\frac{19}{2}$, so we have

$$
\left(\frac{(d+7)^{2}}{2}+3\left(6 d+\frac{19}{2}\right)\right)-6 d-\frac{27}{2} \leqslant 6(d+7) \sqrt{6 d+\frac{19}{2}},
$$

or

$$
\frac{d^{2}}{2}+19 d+\frac{79}{2} \leqslant 6(d+7) \sqrt{6 d+\frac{19}{2}} .
$$

Multiplying by 2,

$$
d^{2}+38 d+79 \leqslant 12(d+7) \sqrt{6 d+\frac{19}{2}} .
$$


Squaring and clearing the right hand side,

$$
d^{4}-788 d^{3}-11862 d^{2}-55484 d-60791 \leqslant 0,
$$

meaning $d \leqslant 802$ in this case as well.

Therefore, if $n<\frac{d^{2}}{2}-1$, it follows that $d \leqslant 802$, so if $d \geqslant 803$, then $n \geqslant \frac{d^{2}}{2}-1$. Substituting back in $d=n-k+2$ and solving the quadratic (and adding the obvious assumption $n \geqslant k$ ), we obtain that $n \leqslant k+\lfloor\sqrt{2 k-1}\rfloor-1$ if $n-k \geqslant 801$.

Thus, if $n>k+\lfloor\sqrt{2 k-1}\rfloor-1$, then $801>n-k>\lfloor\sqrt{2 k-1}\rfloor-1$, so $n-k \leqslant 800$ and so $\sqrt{2 k-1}<801$. Thus $2 k-1<641601$, so $k \leqslant 320800$.

Therefore, if $k \geqslant 320801$, there is no $k$-separator of length greater than $k+\lfloor\sqrt{2 k-1}\rfloor-$ 1 , as desired.

\section{Acknowledgements}

This research was conducted at the University of Minnesota Duluth REU program, supported by NSF/DMS grant 1062709 and NSA grant H98230-11-1-0224.

I would especially like to thank Joe Gallian, the director of the Duluth REU program, for providing the opportunity for this research, suggesting an interesting and relevant problem for me to work on, and his help throughout the writing process.

I would also like to thank the advisors, Sam Elder and Krishanu Sankar, as well as the numerous visitors over the course of the program.

Finally, I would like to thank the reviewer, who provided numerous comments that improved the clarity of the paper.

\section{References}

[1] R. Arratia, On the Stanley-Wilf Conjecture for the Number of Permutations Avoiding a Given Pattern, Electronic Journal of Combinatorics, Volume 6 (1999).

[2] P. Hegarty, Permutations All of Whose Patterns of a Given Length are Distinct, Journal of Combinatorial Theory, Series A, Vol. 120, Issue 7, 1663-1671 (2013).

[3] D. Knuth, The Art of Computer Programming Vol. 1, Addison-Wesley, Boston, 1968.

[4] A. Miller, Asymptotic Bounds for Permutations Containing Many Different Patterns, Journal of Combinatorial Theory, Series A, Volume 119, Issue 1, 92-108 (2009). 